\title{
Agro-Industrial Waste Valorization: Transformation of Starch from Mango Kernel into Biocompatible, Thermoresponsive and High Swelling Nanogels
}

\author{
Marlizia A. L. de Oliveira, ${ }^{a}$ Liszt Y. C. Madruga, ${ }^{\circ}{ }^{b}$ Bruna L. B. de Lima, ${ }^{a}$ \\ Marcos A. Villetti, ${ }^{c}$ Men S. M. de Souza Filho, ${ }^{d}$ Matt J. Kipper, ${ }^{b, e}$ \\ Nivia N. Marques ${ }^{a}$ and Rosangela C. Balaban ${ }^{\circledR *, a}$ \\ ${ }^{a}$ Instituto de Química, Universidade Federal do Rio Grande do Norte, 59078-970 Natal-RN, Brazil \\ ${ }^{b}$ Department of Chemical and Biological Engineering, Colorado State University, \\ 80523-1301 Fort Collins-CO, United States \\ 'Laboratório de Espectroscopia e Polímeros (LEPOL), Universidade Federal de Santa Maria, \\ 97105-900 Santa Maria-RS, Brazil \\ ${ }^{d}$ Embrapa Agroindústria Tropical, 60511-110 Fortaleza-CE, Brazil \\ ${ }^{e}$ School of Biomedical Engineering, Colorado State University, 80523-1301 Fort Collins-CO, \\ United States
}

\begin{abstract}
Mango industry processing disposes $40-60 \%$ of this fruit as residues, such as peels and kernels. The exploration of bioproducts from these industrial rejects can reduce environmental impact besides of producing high value-added materials. In this scenario, carboxymethyl starch nanoparticles were produced from mango (Mangifera indica L.) kernel starch. These nanoparticles were then decorated with thermoresponsive chains of the amino terminated poly $\left(N\right.$-isopropylacrylamide) (PNIPAM-NH $\mathrm{N}_{2}$ ), with the intention of evaluating their applicability in the biomedical area. Elemental analysis, Fourier transform infrared (FTIR) and ${ }^{1} \mathrm{H}$ nuclear magnetic resonance (NMR) spectroscopy confirmed successful grafting of PNIPAM-NH $\mathrm{N}_{2}$ onto the carboxymethyl starch backbone. Scanning electron microscopy (SEM) images and dynamic light scattering (DLS) data showed sizes of 100 and $112 \mathrm{~nm}$ in the dry state and of 744 and $598 \mathrm{~nm}$ in the hydrated state, when the grafting degree (GD) was of 6 and $14.3 \%$, respectively. The degree of swelling was of 41,100 and $15,100 \%$ for GD of 6 and $14.3 \%$ respectively, suggesting that the nanogels are suitable for drug incorporation. The toxicity of the nanogels to human adipose-derived stem cells (ADSCs) and red blood cells (RBCs) was evaluated by lactate dehydrogenase (LDH), alamarBlue and hemolysis assays. Both nanogels were non-cytotoxic and non-hemolytic, suggesting the suitability of these biomaterials for cell- and blood-contacting applications.
\end{abstract}

Keywords: nanogel, swelling, cytotoxicity, thermoresponsive, starch

\section{Introduction}

The use of polysaccharides as matrices of nanogels in biomedical applications has been increasingly studied, as this strategy adds biologically favorable properties to the resulting materials. ${ }^{1-4}$ Starch, for example, commonly found in wheat, corn, potato and cassava, is one of the polysaccharides most widely used in the pharmaceutical industry.

In recent years, the increasing demand for starch in food and non-food related applications has encouraged

*e-mail: rosangelabalaban@hotmail.com the development of unconventional starch sources, such as fruit wastes. Mangoes exportation in 2019 reached approximately 1531,594 tons worldwide. It is estimated that $40-60 \%$ of mango production is rejected as waste, in forms such as peels and kernels. Almost $60 \%$ of the mango kernel is due to starch on a dry weight basis. Then, mango kernel constitutes a potential source of value-added products from starch extraction. ${ }^{5-8}$

The introduction of thermoresponsive polymers with a lower critical solution (LCST) onto the polysaccharide structure promotes the formation of derivatives with improved properties when compared to unmodified 
starch. ${ }^{5,9,10}$ Poly ( $N$-isopropylacrylamide) (PNIPAM) is the most studied thermoresponsive polymer, with a LCST at about $32{ }^{\circ} \mathrm{C}$ in water. Nanogels with PNIPAM have found broad biomedical applications, including cell encapsulation and transplantation, wound healing and drug delivery. ${ }^{11-15}$

Some studies ${ }^{16-18}$ have reported the combination of starch with PNIPAM, showing nanogels with improved mechanical resistance and a degree of swelling up to $1,826 \%$. However, expensive and/or complex routes were employed, such as polymerization by using supercritical carbon dioxide and single electron transfer living radical polymerization (SET-LRP). In addition, no studies were found on the biocompatibility investigation of these nanogels.

Herein is proposed a simple and effective synthetic route to obtain mango starch nanogels decorated with PNIPAM chains, by a coupling reaction. The degree of swelling, thermoresponsive properties and the biocompatibility of the nanogels towards human adipose-derived stem cells (ADSCs) and red blood cells (RBCs) were investigated.

\section{Experimental}

\section{Materials}

Starch extracted from mango (Mangifera indica L., variety Tommy Atkins) kernel (SisGen: AD3DC70) (Natal, Brazil) was purified and characterized previously as having an amylose/amylopectin content of 25/75 wt.\% and a molecular weight $\left(\mathrm{M}_{\mathrm{w}}\right)=3.6 \times 10^{6} \mathrm{~g} \mathrm{~mol}^{-1}$ by gel permeation chromatography (GPC). ${ }^{19,20}$ $N$-Isopropylacrylamide (NIPAM), $N$-hydroxysuccinimide (NHS), 2-aminoethanethiol hydrochloride (AET.HCl) and 1-ethyl-3-[3-(dimethylamino)propyl] carbodiimide hydrochloride (EDC) were supplied by Sigma-Aldrich (São Paulo, Brazil). Dimethylsulfoxide (DMSO), monochloroacetic acid $\left(\mathrm{ClCH}_{2} \mathrm{COOH}\right)$ and isopropyl alcohol were obtained from Synth (São Paulo, Brazil). Hydrochloric acid $32 \% \mathrm{~m} / \mathrm{m}(\mathrm{HCl})$ was supplied by Proquímios (Rio de Janeiro, Brazil). Sodium hydroxide $(\mathrm{NaOH})$ was obtained from Isofar (Duque de Caxias, Brazil). Glutaraldehyde was acquired from Riedel-de Haën (Seelze, Germany) and potassium persulfate (KPS) was obtained from Vetec (São Paulo, Brazil). All the chemicals were used without further purification.

\section{Synthesis of starch-based nanogel}

Starch $(0.9 \mathrm{~g})$ was added to $40 \mathrm{~mL}$ of DMSO/water (75/25) and left under magnetic stirring for $24 \mathrm{~h}$, at room temperature. The mixture was then heated to $60{ }^{\circ} \mathrm{C}$ and $1 \mathrm{~mL}$ of $1 \mathrm{M} \mathrm{HCl}$ was added to the system. After $30 \mathrm{~min}$, glutaraldehyde $(0.1 \mathrm{~g})$ was added and the reaction ${ }^{21}$ was left to proceed for $2 \mathrm{~h}$, at $60{ }^{\circ} \mathrm{C}$, under mechanical stirring. After this time, $100 \mathrm{~mL}$ of isopropyl alcohol was added to quench the reaction. The precipitate was washed with isopropyl alcohol/water (80/20) and then with pure isopropyl alcohol. The product was dried in an oven, at $60{ }^{\circ} \mathrm{C}$, for $24 \mathrm{~h}$.

\section{Synthesis of carboxymethyl starch (CMS)}

Starch-based nanogel $(1 \mathrm{~g})$ was added to $30 \mathrm{~mL}$ of DMSO and left under magnetic stirring, at room temperature, for $24 \mathrm{~h}$. Then, $20 \mathrm{~mL}$ of $5 \mathrm{M} \mathrm{NaOH}$ was added, and the system was heated to $70{ }^{\circ} \mathrm{C}$. After $30 \mathrm{~min}$, monochloroacetic acid $(7 \mathrm{~g})$ was added and the reaction proceeded for $3 \mathrm{~h}$, under mechanical stirring. The product was purified through dialysis against distilled water until the conductivity was close to $10 \mu \mathrm{S} \mathrm{cm}$. Finally, carboxymethylated starch (CMS) was recovered by freeze-drying. A carboxymethylation degree (CD) of $29 \%$ was determined by potentiometric titration, following the methodology described elsewhere. ${ }^{22}$

\section{Synthesis of PNIPAM-NH ${ }_{2}$}

The amino-terminated poly ( $N$-isopropylacrylamide) (PNIPAM-NH ${ }_{2}$ ) was prepared by radical polymerization, using the redox couple APS/AET.HCl as initiators, inside of a glove box $\left(\mathrm{N}_{2}\right.$ gas), according to the procedure described previously by our research group. ${ }^{23}$ Total amino content of $6.0 \times 10^{-4} \mathrm{~mol} \mathrm{~g}^{-1}$ on PNIPAM-NH ${ }_{2}$ was determined by potentiometric titration.

\section{Synthesis of CMS-g-PNIPAM}

Carboxymethylated starch (CMS) was added to $150 \mathrm{~mL}$ of distilled water and left under magnetic stirring, for $24 \mathrm{~h}$. Separately, PNIPAM- $\mathrm{NH}_{2}$ was solubilized in $150 \mathrm{~mL}$ of distilled water, for $30 \mathrm{~min}$. The two solutions were then combined and homogenized under magnetic stirring. After $40 \mathrm{~min}$, NHS and EDC were respectively added and the reaction proceeded for $24 \mathrm{~h}$, at room temperature $\left(\mathrm{ca} .25^{\circ} \mathrm{C}\right.$ ) (Table 1). At the end of the reaction, the $\mathrm{pH}$ of the mixture was adjusted to 2. After that, $\mathrm{NaCl}$ and isopropyl alcohol were added to the system, until precipitation of the product was obtained. The supernatant was withdrawn from the vessel and the precipitate was dialyzed against distilled water until the conductivity was close to $10 \mu \mathrm{S} \mathrm{cm}^{-1}$. The material was recovered by freeze-drying. 
Table 1. Feed reactant concentrations for the grafting reactions and their respective grafting degrees (GD)

\begin{tabular}{lcccccc}
\hline Code & $-\mathrm{COOH} /\left(\mathrm{mol} \mathrm{L}^{-1}\right)$ & $-\mathrm{NH}_{2} /\left(\mathrm{mol} \mathrm{L}^{-1}\right)$ & $\mathrm{EDC} /\left(\mathrm{mol} \mathrm{L}^{-1}\right)$ & $\mathrm{NHS} /\left(\mathrm{mol} \mathrm{L}^{-1}\right)$ & $\mathrm{GD}^{\mathrm{a}} / \%$ & $\begin{array}{c}\text { Grafting efficiency } \\
/ \%\end{array}$ \\
\hline CMS- -PNIPAM1 & 1 & 1 & 4 & 2 & 6.0 & 20.7 \\
CMS-g-PNIPAM2 & 1 & 2 & 4 & 2 & 14.3 & 49.3 \\
\hline
\end{tabular}

${ }^{a}$ The grafting degree $(\mathrm{GD})$ was determined by ${ }^{1} \mathrm{H}$ NMR from equation 1: GD $(\%)=\left(\mathrm{I}_{\mathrm{CH} 3} / 6 \mathrm{I}_{\mathrm{Hanom}}\right) \times 100$. EDC: 1-ethyl-3-[3-(dimethylamino)propyl] carbodiimide hydrochloride; NHS: $N$-hydroxysuccinimide.

\section{Characterization}

\section{Elemental analysis}

Elemental analysis was performed using a EuroEA Elemental Analyser from EuroVector (Pavia, Italy). A sample mass of about $1 \mathrm{mg}$ was used in each analysis. The sample was burned at $980{ }^{\circ} \mathrm{C}$, using oxygen as the combustion gas and helium as the mobile gas.

\section{Fourier-transform infrared spectroscopy (FTIR)}

Infrared spectra were obtained using an FTIR model IRAffinity-1 spectrophotometer from Shimadzu (Kyoto, Japan). Spectra were analyzed with an attenuated total reflectance (ATR) accessory and recorded at a spectral range between 500 and $4000 \mathrm{~cm}^{-1}$, with a resolution of $4 \mathrm{~cm}^{-1}$ and 34 scans.

\section{Proton nuclear magnetic resonance ( ${ }^{1} \mathrm{H}$ NMR)}

${ }^{1} \mathrm{H}$ NMR spectra were obtained with a $600 \mathrm{MHz}$ BioSpin spectrometer from Bruker (Massachusetts, USA), at $60{ }^{\circ} \mathrm{C}$, using DMSO- $d_{6}$ as the solvent. The samples were prepared at a polymer concentration of $20 \mathrm{~g} \mathrm{~L}^{-1}$ and the chemical shifts were reported in ppm and calibrated against tetramethylsilane (TMS) as standard.

The integration of the characteristic peaks of PNIPAM$\mathrm{NH}_{2}$ (methyl protons) and CMS (anomeric proton) on the spectra of the graft copolymers were employed to calculate the grafting degree (GD), according to equation 1. ${ }^{3,24}$

$\mathrm{GD}(\%)=\frac{\mathrm{I}_{\mathrm{CH}_{3}}}{6 \mathrm{I}_{\mathrm{H}_{\text {anom }}}} \times 100$

where $\mathrm{I}_{\mathrm{CH}_{3}}$ is the integration value of the methyl protons and $\mathrm{I}_{\mathrm{H}_{\text {anom }}}$ the integration value of the anomeric proton. The $\% \mathrm{GD}$ represents the average number of PNIPAM chains grafted per 100 anhydroglucose units.

\section{Ultraviolet-visible (UV-Vis)}

To evaluate the thermoresponsive behavior of the samples in water, transmittance measurements were performed on a Cary 50 Bio UV-Vis spectrophotometer from Varian (Palo Alto, USA). The equipment operated at a wavelength of $600 \mathrm{~nm}$, on a temperature range of $25-40^{\circ} \mathrm{C}$, at a polymer concentration of $5 \mathrm{~g} \mathrm{~L}^{-1}$.

\section{Dynamic light scattering (DLS) and zeta potential}

Dynamic light scattering and zeta potential analyses were performed on a Brookhaven ZetaPlus instrument (New York, USA), at $25{ }^{\circ} \mathrm{C}$. CMS and CMS- $g$-PNIPAM copolymers were dispersed in water, at a concentration of $1 \mathrm{~g} \mathrm{~L}^{-1}$. The diameter of the samples was measured at a fixed angle of $90^{\circ}$.

\section{Scanning electron microscopy (SEM)}

The morphology of the nanogels was investigated by scanning electron microscopy (SEM). One drop of the aqueous suspension of the nanogel $\left(0.33 \mathrm{~g} \mathrm{~L}^{-1}\right)$ was deposited on a silicate plate and dried in a desiccator at $25^{\circ} \mathrm{C}$ for $24 \mathrm{~h} .{ }^{25}$ The plate was then coated with gold and analyzed by field emission scanning electron microscopy (FE-SEM) on an Auriga (New York, USA) model microscope from Carl Zeiss.

\section{Swelling behavior}

The swelling degree (SW) was determined by using equation $2: 26-28$

$$
\operatorname{SW}(\%)=100 \times\left[\left(\mathrm{d}_{\text {hyd }} / \mathrm{d}_{\mathrm{dry}}\right)^{3}-1\right]
$$

where $d_{\text {hyd }}$ represents the hydrated particle diameter obtained from DLS measurement and $\mathrm{d}_{\text {dry }}$ corresponds to the dried particle diameter obtained from SEM.

\section{In vitro tests}

For in vitro tests using human materials, a previous consent has been obtained from all subjects.

\section{Cell culture}

Human adipose-derived stem cells (ADSC) were isolated by Prof Kimberly Cox-York of the Department of Food Science and Human Nutrition (Colorado State University) from abdominal and femoral subcutaneous adipose tissue biopsies. The protocol for ADSC isolation from healthy individuals was approved by Colorado State University 
Institutional Review Board. ${ }^{29}$ The cells were cultured at $37{ }^{\circ} \mathrm{C}$, in a $5 \% \mathrm{CO}_{2}$ atmosphere, in $175 \mathrm{~cm}^{2}$ surface area tissue-culture polystyrene flasks, using a growth media (MEM Alpha Modification, HyCloneTM, with 10\% fetal bovine serum and $1 \%$ penicillin/streptomycin added). All ADSC used in this study were below passage five. Before cell seeding, nanogels were dispersed in growth media and filtered using $0.22 \mu \mathrm{m}$ membrane filters. The cells were seeded into nanogel dispersions at a concentration of 10,000 cells per well in 48-well plates, and they were cultured at $37{ }^{\circ} \mathrm{C}$ in a $5 \% \mathrm{CO}_{2}$ atmosphere. ${ }^{29}$ All tests were performed in at least triplicate or quadruplicate $(\mathrm{n}=3$ or $n=4$ ). Cells cultured on tissue-culture polystyrene was used as controls in all assays.

\section{Cytotoxicity}

The lactate dehydrogenase (LDH) assay was performed after 1 day of culture to evaluate the toxicity of the nanogels towards the cells. After $24 \mathrm{~h}$ of cell culture in media containing the nanogel samples, $50 \mu \mathrm{L}$ of media with the samples was added to an equal amount of LDH (Quantichrom ${ }^{\mathrm{TM}}$ BioAssay Systems, CA, USA) in a 96-well plate, followed by an incubation of $30 \mathrm{~min}$. The absorbance of the solution in each well was measured at 490 and $680 \mathrm{~nm}(\mathrm{n}=4)$, using a plate reader (BMG LABTECH FLUOstarOmega, Ortenburg, Germany). ${ }^{30}$

\section{Cell viability}

Cell viability was determined by alamarBlue ${ }^{\circledR}$ assay (Promega G808A, Madison, WI, USA). ${ }^{31}$ Nanogel dispersions were prepared in serum-supplemented tissue culture medium and sterilized by filtration $(0.22 \mu \mathrm{m}$, Schleicher \& Schuell, Dassel, Germany). After 4 and 7 days, $50 \mu \mathrm{L}$ of CellTiter-Blue dye was added to $500 \mu \mathrm{L}$ of the sample in the culture media. After $8 \mathrm{~h}$ incubation at $37{ }^{\circ} \mathrm{C}$ in $5 \% \mathrm{CO}_{2}$ atmosphere, the absorbance of each sample was read in a microplate reader (Molecular Devices Spectra Max M3, Sunnyvale, CA, USA) at 570 and $600 \mathrm{~nm}$ to evaluate cell viability.

\section{Hemolytic activity}

The hemolytic activity of the polymers was determined. ${ }^{31}$ $8 \mathrm{~mL}$ of human blood, collected from a healthy donor in heparinized tubes were centrifuged at $700 \mathrm{~g}$, for $10 \mathrm{~min}$. Whole human blood was acquired through venipuncture from healthy individuals, and formal assents were obtained from the donors. Colorado State University Institutional Review Board approved the protocol for blood isolation from healthy participants. All experiments were conducted in agreement with the National Institutes of Health's "Guiding Principles for Ethical Research". ${ }^{2,4}$ The plasma was removed and the pellet was washed three times with cold phosphate buffer solution (PBS, $\mathrm{pH}$ 7.4) by centrifugation at $700 \mathrm{~g}$, for $10 \mathrm{~min}$, and resuspended in $5 \mathrm{~mL}$ of the same buffer. $80 \mu \mathrm{L}$ of red blood cells (RBC) suspension were added to $4 \mathrm{~mL}$ of nanogel dispersions of 0.5 or $1.0 \mathrm{mg} \mathrm{mL}^{-1}$, also prepared in PBS buffer, and were incubated for $60 \mathrm{~min}$, at $37^{\circ} \mathrm{C}$, in a shaker. The release of hemoglobin was determined, after centrifugation, by photometric analysis of the supernatant at $540 \mathrm{~nm}$. Complete hemolysis was achieved using $0.2 \%$ Triton X-100 yielding the $100 \%$ control value. The experiments were run in triplicate $(\mathrm{n}=3)$.

\section{Statistical analysis}

All the experiments were carried out on at least triplicate and the results are presented as mean \pm standard deviation. The statistical differences were determined using one-way analysis of variance (ANOVA) analysis $(P<0.05)$ with a post-hoc Tukey's HSD (honest significant difference) test.

\section{Results and Discussion}

\section{Elemental analysis}

Figure 1 shows the weight percentages of $\mathrm{C}, \mathrm{H}, \mathrm{O}$ and $\mathrm{N}$ on the samples, determined by elemental analysis. As expected, neither starch, crosslinked starch, nor CMS had nitrogen. The introduction of PNIPAM chains onto CMS was indicated by the presence of nitrogen in CMS- $g$ PNIPAM1 and CMS- $g$-PNIPAM2. ${ }^{32}$ Also, with the increase in the feed amount of PNIPAM-NH${ }_{2}$, the percentages of $\mathrm{C}, \mathrm{H}$ and $\mathrm{N}$ were increased, suggesting a higher grafting degree for CMS- $g$-PNIPAM2 than for CMS- $g$-PNIPAM1.

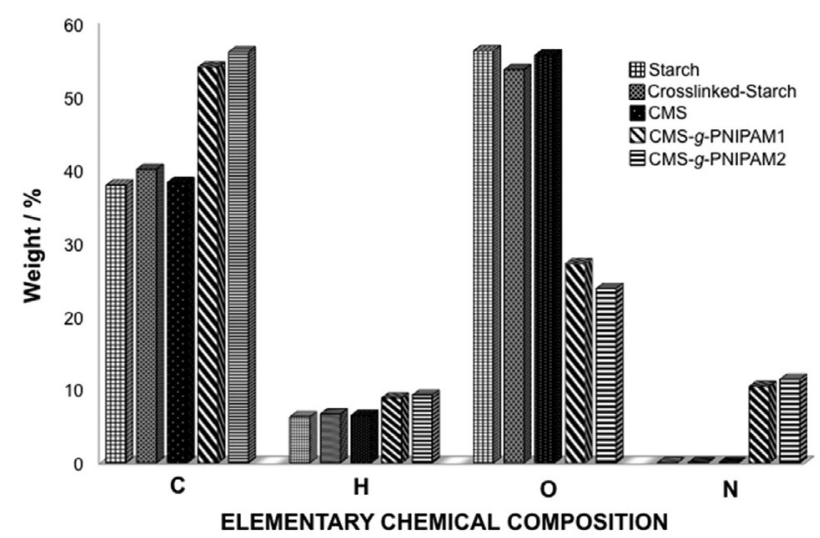

Figure 1. Weight percentages of $\mathrm{C}, \mathrm{H}$, and $\mathrm{N}$ atoms in starch, crosslinkedstarch, CMS, PNIPAM-NH 2 , CMS- $g$-PNIPAM1 and CMS- $g$-PNIPAM2.

\section{Fourier-transform infrared spectroscopy (FTIR)}

The FTIR spectra of starch and its derivatives (crosslinkedstarch, CMS, CMS-g-PNIPAM1 and CMS- $g$-PNIPAM2) 
(Figure 2) exhibit a peak at $3330 \mathrm{~cm}^{-1}$, attributed to $\mathrm{O}-\mathrm{H}$ stretching vibrations; a peak at $2920 \mathrm{~cm}^{-1}$, corresponding to $\mathrm{C}-\mathrm{H}$ stretching and peak at around $1015 \mathrm{~cm}^{-1}$, ascribed to $\mathrm{C}-\mathrm{O}$ stretching of the anydroglucose units. ${ }^{33,34}$ The CMS spectrum also shows peaks at 1580 and $1392 \mathrm{~cm}^{-1}$, which can be attributed to the asymmetric symmetric stretch of $\mathrm{COO}^{-}$, respectively, revealing the success of the carboxymethylation on crosslinked-starch. ${ }^{35,36}$

In addition to the aforementioned starch peaks, the FTIR spectra of the copolymers also exhibit the characteristic peaks of PNIPAM: at $1626 \mathrm{~cm}^{-1}$, due to $\mathrm{C}=\mathrm{O}$ stretching (amide I); at $1530 \mathrm{~cm}^{-1}$, ascribed to $\mathrm{N}-\mathrm{H}$ stretching (amide II); and at 1371 and $1366 \mathrm{~cm}^{-1}$, corresponding to the symmetric bend (or umbrella bend) of isopropyl groups. These peaks indicate that the introduction of PNIPAM grafts onto CMS was successful. ${ }^{37,38}$ (a)

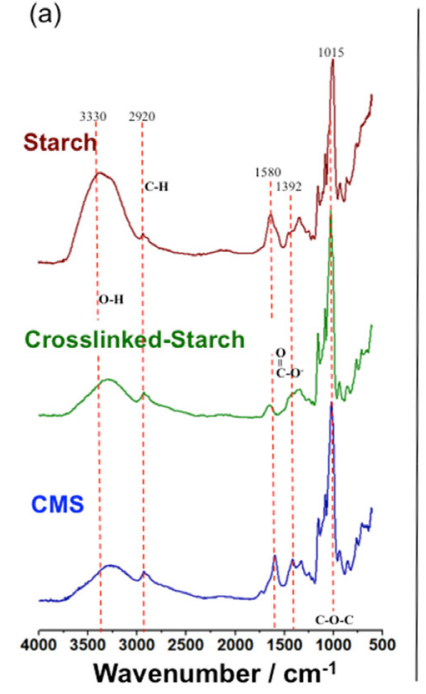

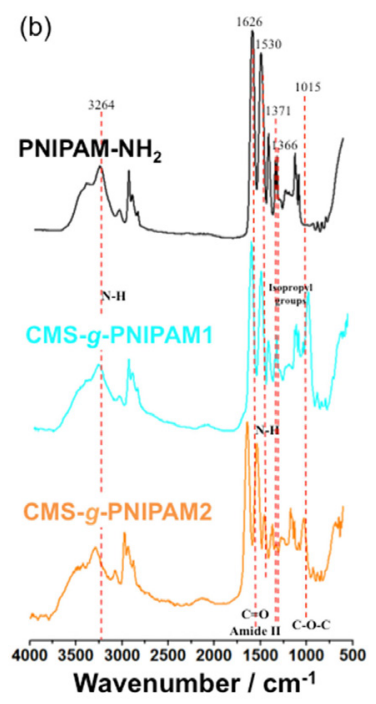

Figure 2. FTIR (ATR) spectra of (a) starch, crosslinked-starch, and CMS, (b) PNIPAM-NH ${ }_{2}$, CMS- $g$-PNIPAM1 and CMS- $g$-PNIPAM2.

\section{Proton nuclear magnetic resonance ( ${ }^{1} \mathrm{H}$ NMR)}

The ${ }^{1} \mathrm{H}$ NMR spectrum of CMS (Figure 3) exhibits peaks at 5.0-5.5 ppm, attributed to anomeric protons $\left(1,1^{\prime}\right)$ from alpha 1-4 and alpha 1-6 glycosidic carbon bonds. The other hydrogens appeared at the 3.0-4.5 ppm region. ${ }^{39-41}$

The ${ }^{1} \mathrm{HNMR}$ spectrum of PNIPAM- $\mathrm{NH}_{2}$ shows an intense peak at $1.06 \mathrm{ppm}$, attributed to the methyl groups (a). The methylene and methinic hydrogens (b and c) are observed at 1.45 and $1.99 \mathrm{ppm}$, respectively. The hydrogen $\mathrm{d}$ is observed at $3.85 \mathrm{ppm}$ and the $\mathrm{N}-\mathrm{H}$ appears at $6.98 \mathrm{ppm}$ (e). ${ }^{17}$

CMS- $g$-PNIPAM1 displays the characteristic peaks of both CMS and PNIPAM on ${ }^{1} \mathrm{H}$ NMR spectrum, evidencing the grafting of PNIPAM- $\mathrm{NH}_{2}$ onto carboxymethyl starch. CMS- $g$-PNIPAM2 presents a similar spectral feature (data not shown). The CMS-g-PNIPAM2 copolymer has a GD
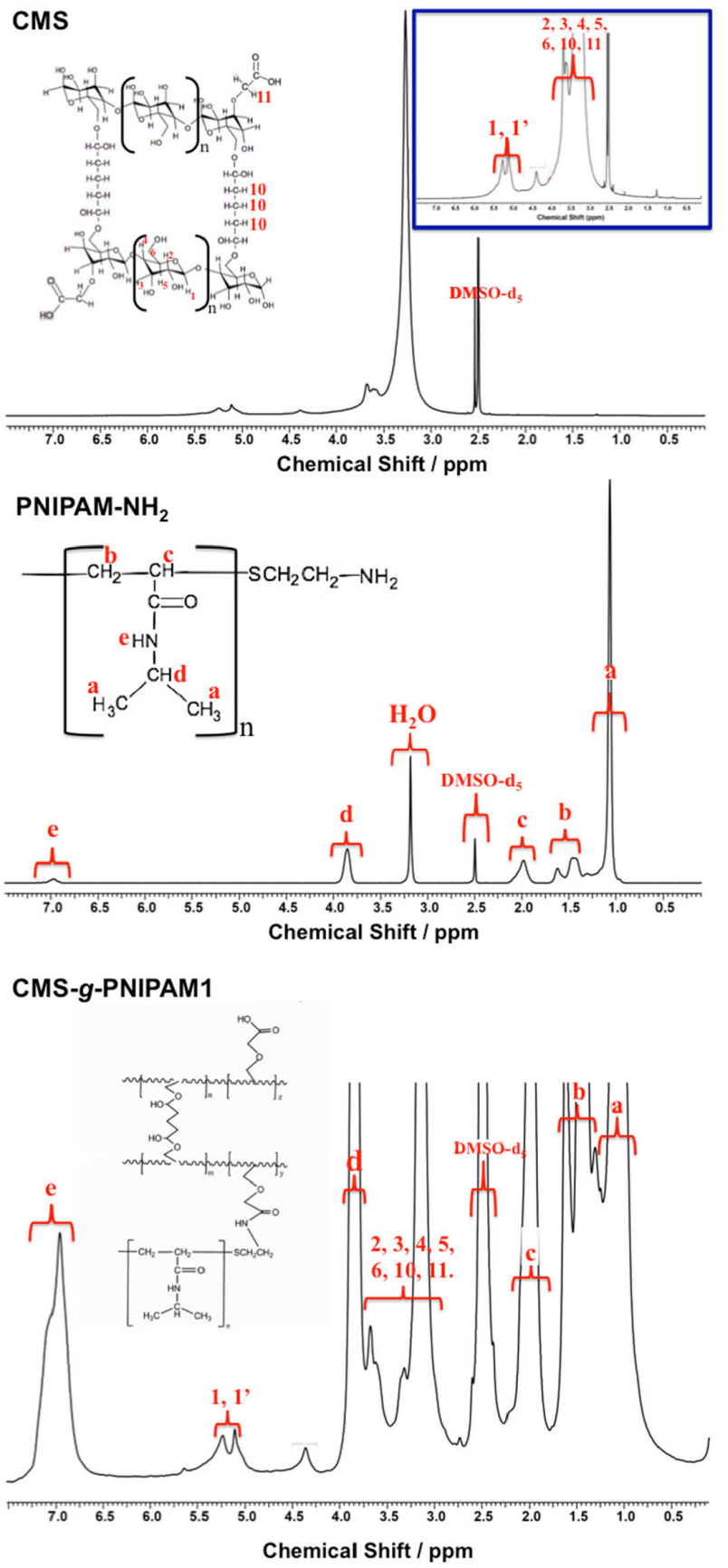

Figure 3. ${ }^{1} \mathrm{H}$ NMR spectra $\left(600 \mathrm{MHz}\right.$, DMSO- $\left.d_{6}\right)$ of CMS, PNIPAM- $\mathrm{NH}_{2}$ and CMS- $g$-PNIPAM1 (with magnification).

of $14.3 \%$, whereas CMS- $g$-PNIPAM1 has a GD of $6 \%$, as determined by equation 1 . This indicates that increasing the feed molar amount of PNIPAM- $\mathrm{NH}_{2}$ during synthesis increases the grafting degree of the copolymer, as also observed by elemental analysis. ${ }^{3,42,43}$

UV-Vis

Figure 4 displays the transmittance versus temperature curves for CMS, PNIPAM- $\mathrm{NH}_{2}$ and their derivatives in 

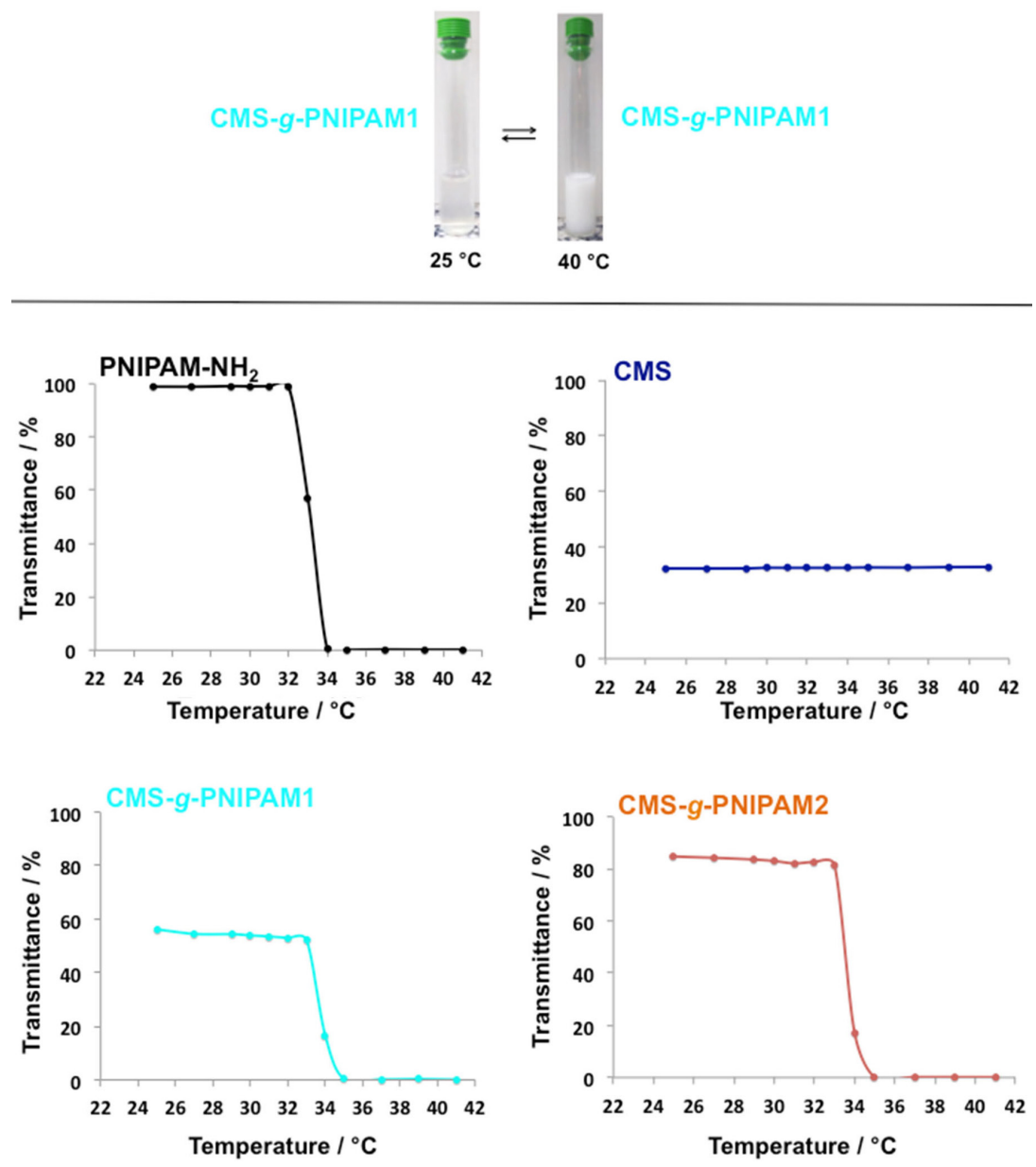

Figure 4. Thermoresponsive behavior in water of CMS, PNIPAM-NH ${ }_{2}$, CMS- $g$-PNIPAM1 and CMS- $g$-PNIPAM2 and the visual appearance of the dispersion of CMS- $g$-PNIPAM1 before and after heating.

water, at a polymer concentration of $5 \mathrm{~g} \mathrm{~L}^{-1}$. As expected, CMS did not exhibit a thermoresponsive behavior in water and the transmittance remained constant throughout the $25-40{ }^{\circ} \mathrm{C}$ heating process. On the other hand, PNIPAM, as a thermoresponsive polymer, has a cloud point temperature (Tcp) of $33^{\circ} \mathrm{C}$. The initial transmittance of CMS was lower than the one of PNIPAM- $\mathrm{NH}_{2}$, because CMS particles only swell in water, whereas PNIPAM- $\mathrm{NH}_{2}$ chains are highly soluble in the solvent. For this reason, the introduction of the thermoresponsive chains onto CMS particles makes them more translucent in water.

It can be seen from Figure 4 that the copolymers have a Tcp at $35^{\circ} \mathrm{C}$, from which the copolymers precipitate. As temperature rises, the grafted PNIPAM chains dehydrate and polymer-solvent hydrogen bonds decrease, while polymer-polymer associations become stronger, leading the particles to collapse. ${ }^{44,45}$

\section{Dynamic light scattering (DLS) and zeta potential}

Table 2 presents zeta potential and the diameter of
CMS, CMS- $g$-PNIPAM1 and CMS- $g$-PNIPAM2 in water. CMS exhibits a negative charge of $-28.3 \mathrm{mV}$, due to the presence of the $\mathrm{COO}^{-}$groups in water. The introduction of PNIPAM chains onto CMS reduces the zeta potential, due to the grafting reaction on the carboxymethyl groups of CMS. Also, as expected, the copolymer with a higher grafting degree has the lower zeta potential.

The effective diameter of carboxymethyl starch in water is $496 \mathrm{~nm}$, which increases after the particles are decorated with PNIPAM chains. The higher diameter of CMS- $g$-PNIPAM1, when compared to CMS- $g$-PNIPAM2 is attributed to the higher amount of $\mathrm{COO}^{-}$groups on CMS-g-PNIPAM1, which promotes a higher swelling in water.

Scanning electron microscopy (SEM)

Figure 5 shows the morphology of the copolymers on a dried state. CMS- $g$-PNIPAM1 and CMS- $g$-PNIPAM2 exhibit a globular structure, with sizes of 100 and $112 \mathrm{~nm}$, respectively. These are much smaller than the diameters 
Table 2. Zeta potential and diameter of CMS, CMS- $g$-PNIPAM1 and CMS- $g$-PNIPAM2 in water

\begin{tabular}{lcc}
\hline Code & Zeta potential / mV & Diameter / nm \\
\hline CMS & $-28.3 \pm 1.8$ & $496 \pm 18$ \\
CMS- $g$-PNIPAM1 & $-16.9 \pm 0.2$ & $744 \pm 27$ \\
CMS- $g$-PNIPAM2 & $-13.2 \pm 0.4$ & $598 \pm 8.7$ \\
\hline
\end{tabular}

CMS: carboxymethyl starch.

obtained by DLS, for the swollen state. From these data, it is possible to determine a degree of swelling (SW) of 41,100 and $15,100 \%$ for CMS- $g$-PNIPAM1 and CMS- $g$-PNIPAM2, respectively. The higher SW of CMS- $g$-PNIPAM1 occurs due to its higher zeta potential $(-16.9 \pm 0.2)$, that promotes greater water absorption.
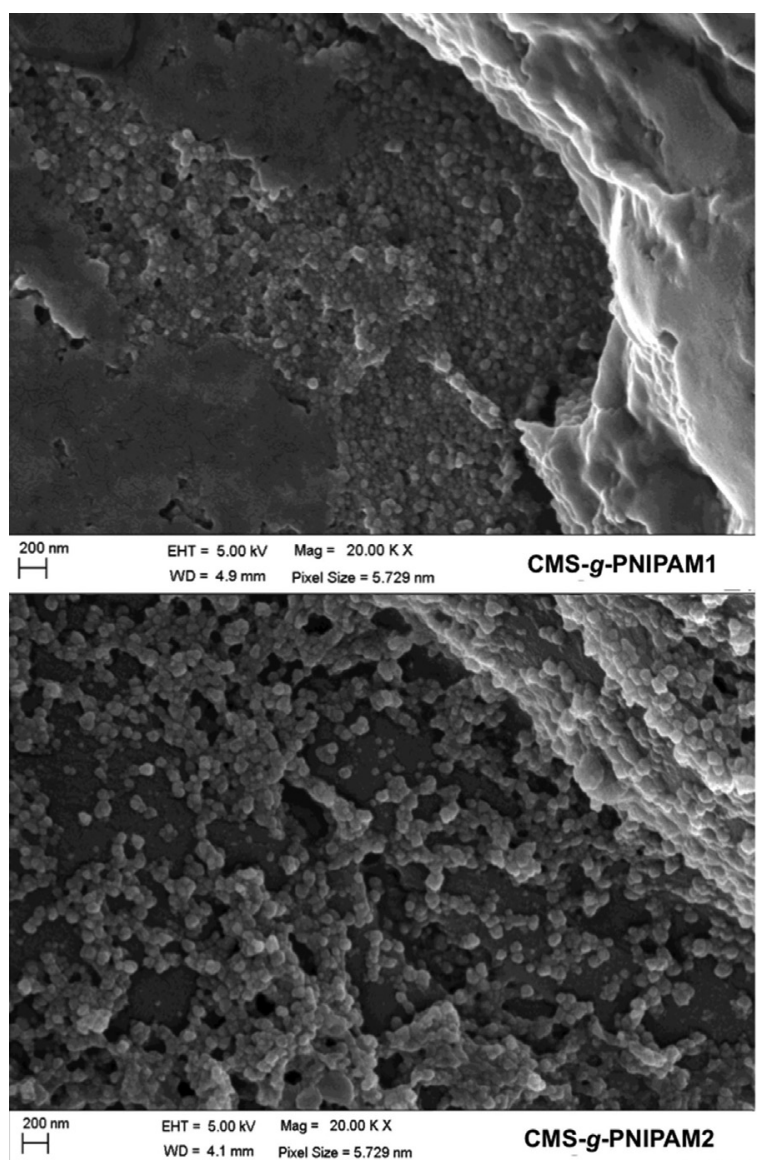

Figure 5. Micrographs obtained by FE-SEM with a magnification of 20,000 times of CMS- $g$-PNIPAM1 and CMS- $g$-PNIPAM2.

The high degree of swelling suggests that the nanogels are suitable for drug incorporation. At human body temperature, hydrophobic molecules would remain trapped inside the gel by hydrophobic associations with the isopropyl groups of PNIPAM. Hydrophilic molecules would make hydrogens bonds with the stiff polysaccharide core of the nanogels while the PNIPAM shell undergoes a temperature triggered contraction, trapping the small molecules.

\section{In vitro tests}

Human adipose-derived stem cells (ADSCs) were chosen for biocompatibility testing because adipose tissue is an abundant and accessible source of stem cells. While in the bone marrow the proportion is approximately 1 stem cell for every 25,000 , in adipose tissue the stem cells it corresponds to approximately $2 \%$ of the total resident cells. Human adipose-derived stem cells have been shown to be biologically similar, but not identical, to bone marrow mesenchymal stem cells. Also, human adipose-derived stem cells have been demonstrated to differentiate into a broad spectrum of different cell lineages, such as bone, cartilage, endothelium, liver, and neural cells, which make them a versatile and reliable source.

The cytotoxicity of the nanogels was estimated by the reaction method with the enzyme $\mathrm{LDH}$, to evaluate the number of cells that would lyse or die in the culture media containing the nanogels. Analyzing an aliquot of the culture media containing cells, reacting with a tetrazolium salt and reading at $490 \mathrm{~nm}$, the absorbance values are correlated to cytotoxicity of the material. The LDH enzyme, which is a cytosolic enzyme, only is released to the extracellular medium and can be quantified if the cell was lysed, indicating toxicity of the nanogels. To calculate the cytotoxicity percentage of the nanogels, a control of cells in culture media on polystyrene and lysis buffer was used as $100 \%$ cytotoxicity and negative control with cells in culture media on polystyrene was used to compare the samples. None of the nanogels were considered cytotoxic since there is no statistical difference to the control and none of them presented cytotoxicity percentages greater than $15 \% .{ }^{29} \mathrm{CMS}-g$-PNIPAM2 at $1.0 \mathrm{mg} \mathrm{mL}^{-1}$ has the lowest value of cytotoxicity, which can be correlated to the higher GD of PNIPAM on this nanogel, since this polymer can improve cell compatibility on surfaces. ${ }^{46,47}$

Cell viability of the nanogels was evaluated in vitro by using alamarBlue reagent. Living cells maintain a reducing environment within their cytoplasm and mitochondria, being able to reduce active agent of alamarBlue (resazurin dye), resulting in a quantifiable color change from blue to pink. The number of living cells is proportional to the absorption of the dye. Figure $6 \mathrm{~b}$ shows the reduction percentage of alamarBlue for the cells in media containing the nanogels after 4 and 7 days of cell culture, at polymer concentrations of 0.5 and $1.0 \mathrm{mg} \mathrm{mL}^{-1}$. The alamarBlue reduction percentage was calculated by using negative control values having only the culture medium and the dye. All nanogels have lower 

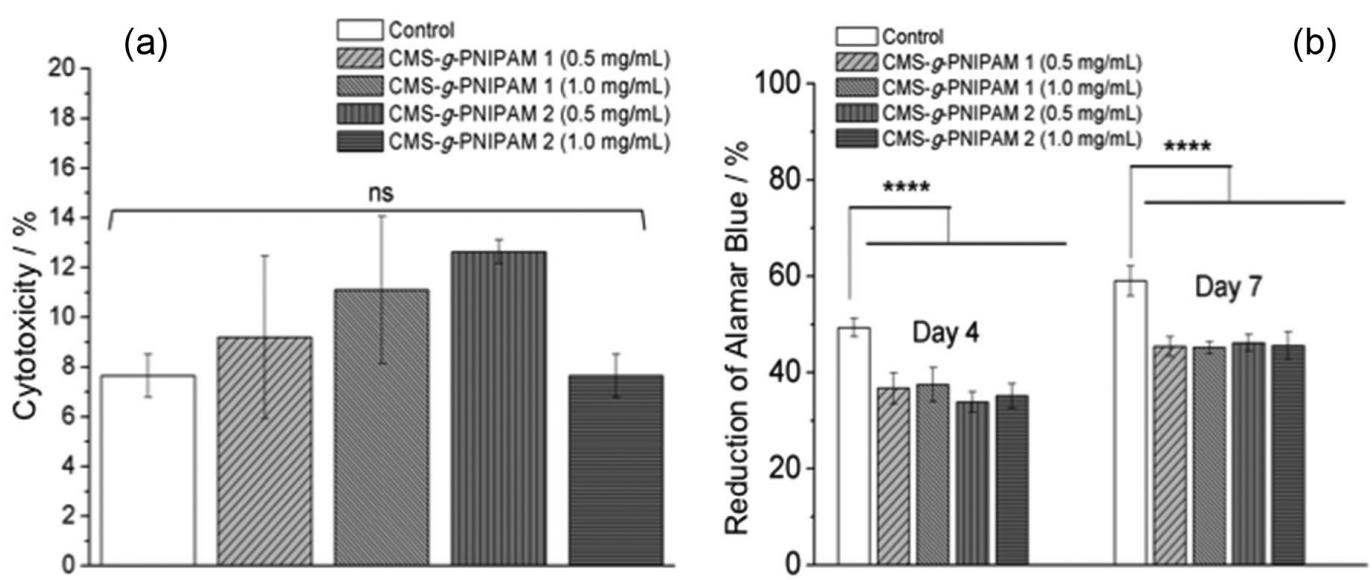

Figure 6. Percentage of cytotoxicity of the nanogels (a) and cell viability after 4 and 7 days of cell culture represented as percentage reduction of alamarBlue (b). Values represent mean \pm standard deviation $(\mathrm{n}=4)$. $* * * * p \leq 0.0001$ and "ns" $p \geq 0.05$, compared to tissue culture polystyrene (control).
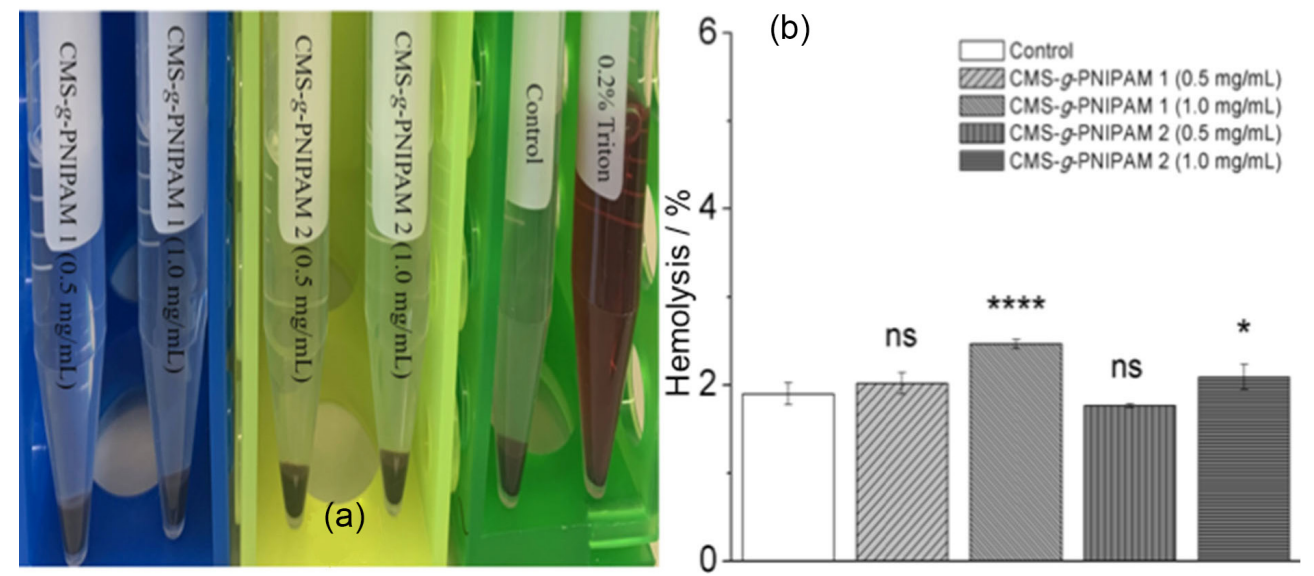

Figure 7. RBCs treated with CMS- $g$-PNIPAM1, CMS- $g$-PNIPAM2, PBS ( $0 \%$ of hemolysis) and Triton X-100 (100\% of hemolysis) (a); percent hemolysis caused by nanogels at concentration of 0.5 and $1.0 \mathrm{mg} \mathrm{mL}^{-1}(\mathrm{~b})$. Values represent mean \pm standard deviation $(\mathrm{n}=3)$. **** $p \leq 0.0001$, * $p \leq 0.05$ and "ns" $p \geq 0.05$, compared to control.

percentages of alamarBlue reduction when compared to the control. However, after 7 days of growth, all experimental samples have about $50 \%$ alamarBlue reduction, indicating that the cells maintain an environment of growth and proliferation..$^{31,48}$ There is no statistical difference between CMS- $g$-PNIPAM1 and CMS- $g$-PNIPAM2 samples, which suggests that both nanogels are non-toxic and can be used for the development of cell contacting materials.

A hemolysis assay is an indispensable initial step in evaluating the blood compatibility of polymeric materials that contact blood to identify severe acute toxic reactions in human red blood cells (RBCs) in vivo..$^{49}$ The hemolysis of RBCs was evaluated after incubation with nanogel suspensions in PBS and compared to Triton X-100 (100\% hemolysis) and to pure PBS (0\% hemolysis) (Figure 7). Cytotoxicity for RBCs is indicated when there is over $10 \%$ hemolysis. ${ }^{31}$ None of the designed nanogels were hemolytic at the concentrations tested, showing less than $4 \%$ hemolysis and clear solutions, with no visible released haemoglobin. ${ }^{50,51}$ This behavior is extremely favorable for blood-contacting applications, like cardiovascular devices, orthopedic implants and drug delivery systems. ${ }^{52-54}$

\section{Conclusions}

In this work, thermoresponsive particles based on mango starch were successfully prepared in water, by using EDC/NHS as coupling agents of the reaction between amino terminated PNIPAM and carboxymethyl starch. Elementary analysis showed nitrogen in the composition of the samples after the coupling reaction, indicating the incorporation of PNIPAM onto the CMS nanogel. The FTIR and ${ }^{1} \mathrm{H}$ NMR spectra of the CMS- $g$-PNIPAM copolymers displayed the characteristic peaks of both CMS and PNIPAM, further evidencing the grafting reaction. The integration of the peaks found in ${ }^{1} \mathrm{H}$ NMR showed a higher grafting degree to the copolymer prepared with a higher amount of PNIPAM-NH ${ }_{2}$ added to the feed. This result was also supported by the zeta potential results. As a result, CMS acquired thermoresponsive properties. 
The high degree of swelling of CMS- $g$-PNIPAM1 $(41,100 \%)$ and CMS- $g$-PNIPAM2 $(15,100 \%)$ suggests a high capacity to incorporate small molecules into the nanogels. Despite of all nanogels had lower percentages of alamarBlue reduction when compared to the control, all experimental samples had about $50 \%$ alamarBlue reduction after 7 days of growth, indicating that the cells maintain an environment of growth and proliferation. Both of them showed no toxicity for ADSCs and non-hemolytic activity for human RBCs, which makes the mango starchbased copolymers non-toxic and suitable materials for contact with ADSCs and RBCs. All of these properties combined make these thermoresponsive particles promising candidates for biomedical applications.

\section{Acknowledgments}

The authors are thankful to Petróleo Brasileiro S.A.Petrobras RJ (SAP 4600580022) and Conselho Nacional de Desenvolvimento Científico e Tecnológico (CNPq grant 304339/2019-9) for their financial support. This study was financed in part by the Coordenação de Aperfeiçoamento de Pessoal de Nível Superior - Brazil (CAPES) - Finance Code 001. Also, the authors gratefully acknowledge the financial support of the Brazilian Agricultural Research Corporation (EMBRAPA, 12.14.04.002.00.00) and from the National Science Foundation (award number 1933552).

\section{Author Contributions}

Marlizia A. L. de Oliveira was responsible for investigation, data curation, formal analysis, writing original draft; Liszt Y. C. Madruga for data curation, formal analysis, writing review and editing; Bruna L. B. de Lima for data curation, formal analysis; Marcos A. Villetti for investigation, formal analysis; Men S. M. de Souza Filho for investigation, conceptualization; Matt J. Kipper for conceptualization, writing-review and editing; Nívia N. Marques for investigation, conceptualization, writing-review and editing; Rosangela C. Balaban for conceptualization, supervision, funding acquisition, project administration, writing-review and editing.

\section{References}

1. Chowhan, A.; Giri, T. K.; Int. J. Biol. Macromol. 2020, 150, 559.

2. Câmara, P. C. F.; Madruga, L. Y. C.; Sabino, R. M.; Vlcek, J.; Balaban, R. C.; Popat, K. C.; Martins, A. F.; Kipper, M. J.; Mater. Sci. Eng., C 2020, 112, 110919.
3. Marques, N. N.; Balaban, R. C.; Halila, S.; Borsali, R.; Carbohydr. Polym. 2018, 184, 108.

4. Sabino, R. M.; Kauk, K.; Madruga, L. Y. C.; Kipper, M. J.; Martins, A. F.; Popat, K. C.; J. Biomed. Mater. Res., Part A 2020, 108, 992.

5. Garcia, M. A. V. T.; Garcia, C. F.; Faraco, A. A. G.; Stärke 2020, 72, 1900270.

6. Marques, N. N.; Garcia, C. S. N.; Madruga, L. Y. C.; Villetti, M. A.; Souza Filho, M. S. M.; Ito, E. N.; Balaban, R. C.; J. Renewable Mater. 2019, 7, 139.

7. Kringel, D. H.; Dias, A. R. G.; Zavareze, E. R.; Gandra, E. A.; Stärke 2020, 72, 1900200.

8. Food and Agriculture Organization of the United Nations (FAO); Major Tropical Fruits - Preliminary Market Results 2019; FAO Fisheries Technical Paper: Rome, Italy, 2020, p. 17.

9. Kavousi, F.; Nikfarjam, N.; Polymer 2019, 180, 121744.

10. Marques, N. N.; Alves, K. S.; Vidal, R. R. L.; Maia, A. M. S.; Madruga, L. Y. C.; Curti, P. S.; Balaban, R. C. In Emerging Research in Science and Engineering Based on Advanced Experimental and Computational Strategies; La Porta, F.; Taft, C., eds.; Springer: Cham, 2020, p. 433.

11. Chandel, A. K. S.; Bera, A.; Nutan, B.; Jewrajka, S. K.; Polymer 2016, 99, 470.

12. Brighenti, R.; Cosma, M. P.; J. Mech. Phys. Solids 2020, 143, 104011.

13. Motlaq, V. F.; Knudsen, K. D.; Nyström, B.; J. Colloid Interface Sci. 2018, 524, 245.

14. Hogan, K. J.; Mikos, A. G.; Polymer 2020, 211, 123063.

15. Razavi, B.; Abdollahi, A.; Roghani-Mamaqani, H.; SalamiKalajahi, M.; Polymer 2020, 187, 122046.

16. Cao, L.; Chen, L.; Chen, X.; Zuo, L.; Li, Z.; Polymer 2006, 47, 4588.

17. Wang, L.; Wu, Y.; Men, Y.; Shen, J.; Liu, Z.; RSC Adv. 2015, 5, 70758.

18. Tan, Y.; Xu, K.; Wang, P.; Li, W.; Sun, S.; Dong, L.; Soft Matter 2010, 6, 1467.

19. Dutra, M.A.L.; Marques, N. N.; Fernandes, R. S.; Souza Filho, M. S. M.; Balaban, R. C.; Ecotoxicol. Environ. Saf. 2020, 200, 110771.

20. Cordeiro, E. M. S.; Nunes, Y. L.; Mattos, A. L. A.; Rosa, M. F.; Macromol. Symp. 2014, 344, 39.

21. El-Tahlawy, K.; Venditti, R. A.; Pawlak, J. J.; Carbohydr. Polym. 2007, 67, 319.

22. Muthukumaran, C.; Kanmani, B. R.; Sharmila, G.; Shanmugaprakash, M. K. N. M.; Carbohydr. Polym. 2018, 194, 311.

23. Marques, N. N.; Lima, B. L. B.; Balaban, R. C.; Macromol. Symp. 2016, 367, 126.

24. Dulong, V.; Mocanu, G.; Picton, L.; Le Cerf, D.; Carbohydr. Polym. 2012, 87, 1522.

25. Chen, J.; Chen, F.; Meng, Y.; Wang, S.; Long, Z.; Polymer 2019 , $168,228$. 
26. Abreu, F. O. M. S.; Bianchini, C.; Forte, M. M. C.; Kist, T. B. L.; Carbohydr. Polym. 2008, 74, 283.

27. Liu, X.; Xue, W.; Liu, Q.; Yu, W.; Fu, Y.; Xiong, X.; Ma, X.; Yuan, Q.; Carbohydr. Polym. 2004, 56, 459.

28. Mousaviasl, S.; Saleh, T.; Shojaosadati, S. A.; Boddohi, S.; Int. J. Biol. Macromol. 2018, 120, 468.

29. Sabino, R. M.; Mondini, G.; Kipper, M. J.; Martins, A. F.; Popat, K. C.; Carbohydr. Polym. 2020, 251, 117079.

30. Madruga, L. Y. C.; Balaban, R. C.; Popat, K. C.; Kipper, M. J.; Macromol. Biosci. 2020, 21, 2000292.

31. Madruga, L. Y. C.; Sabino, R. M.; Santos, E. C. G.; Popat, K. C.; Balaban, R. C.; Kipper, M. J.; Int. J. Biol. Macromol. 2020, 243, 153057.

32. Haroon, M.; Wang, L.; Yu, H.; Ullah, R. S.; Abdin, Z. U.; Khan, R. U.; Chen, Q.; Liu, J.; Carbohydr. Polym. 2018, 186, 150.

33. Iman, M.; Maji, T. K.; Carbohydr. Polym. 2012, 89, 290.

34. Liu, W.; Budtova, T.; Polymer 2012, 53, 5779.

35. Basri, S. N.; Zainuddin, N.; Hashim, K.; Yusof, N. A.; Carbohydr. Polym. 2016, 138, 34.

36. Khushbu; Warkar, S. G.; Kumar, A.; Polymer 2019, 182, 121823.

37. Marques, N. N.; Lima, B. V.; Silveira, V. R.; Lima, B. L. B.; Maia, A. M. S.; Balaban, R. C.; Colloid Polym. Sci. 2016, 294, 981.

38. Gupta, N. R.; Ghute, P. P.; Badiger, M. V.; Carbohydr. Polym. 2011, 83, 74.

39. Chi, H.; Xu, K.; Wu, X.; Chen, Q.; Xue, D.; Song, C.; Zhang, W.; Wang, P.; Food Chem. 2008, 106, 923.

40. Tizzotti, M. J.; Sweedman, M. C.; Tang, D.; Schaefer, C.; Gilbert, R. G.; J. Agric. Food Chem. 2011, 59, 6913.

41. Clasen, S. H.; Müller, C. M. O.; Parize, A. L.; Pires, A. T. N.; Carbohydr. Polym. 2018, 180, 348.

42. Durand, A.; Hourdet, D.; Polymer 1999, 40, 4941.

43. Gupta, N. R.; Torris, A. T. A.; Wadgaonkar, P. P.; Rajamohanan, P. R.; Ducouret, G.; Hourdet, D.; Creton, C.; Badiger, M. V.; Carbohydr. Polym. 2015, 117, 331.
44. Liu, R.; Fraylich, M.; Saunders, B. R.; Colloid Polym. Sci. 2009 287, 627.

45. Recillas, M.; Silva, L. L.; Peniche, C.; Goycoolea, F. M.; Rinaudo, M.; Argüelles-Monal, W. M.; Biomacromolecules 2009, 10, 1633.

46. Schmidt, S.; Zeiser, M.; Hellweg, T.; Duschl, C.; Fery, A.; Möhwald, H.; Adv. Funct. Mater. 2010, 20, 3235.

47. Nash, M. E.; Carroll, W. M.; Nikoloskya, N.; Yang, R.; Connell, C. O.; Gorelov, A. V.; Dockery, P.; Liptrot, C.; Lyng, F. M.; Garcia, A.; Rochev, Y. A.; ACS Appl. Mater. Interfaces 2011, 3, 1980.

48. Pulyala, P.; Singh, A.; Dias-Netipanyj, M. F.; Cogo, S. C.; Santos, L. S.; Soares, P.; Gopal, V.; Suganthan, V.; Manivasagam, G.; Popat, K. C.; Mater. Sci. Eng., C 2017, 75, 1305.

49. Bansal, R.; Pathak, R.; Jha, D.; Kumar, P.; Gautam, H. K.; Int. J. Polym. Mater. Polym. Biomater. 2015, 64, 84.

50. Fischer, D.; Li, Y.; Ahlemeyer, B.; Krieglstein, J.; Kissel, T.; Biomaterials 2003, 24, 1121.

51. Sovadinova, I.; Palermo, E. F.; Huang, R.; Thoma, L. M.; Kuroda, K.; Biomacromolecules 2011, 12, 260.

52. Simon-Walker, R.; Cavicchia, J.; Prawel, D. A.; Dasi, L. P.; James, S. P.; Popat, K. C.; J. Biomed. Mater. Res., Part B 2018, 106, 1964.

53. Riedel, N. A.; Smith, B. S.; Williams, J. D.; Popat, K. C.; Mater. Sci. Eng., C 2012, 32, 1196.

54. Copes, F.; Chevallier, P.; Loy, C.; Pezzoli, D.; Boccafoschi, F.; Mantovani, D.; Front. Bioeng. Biotechnol. 2019, 7, 74.

Submitted: February 16, 2021

Published online: April 26, 2021 